\title{
Cursos MOOC: un enfoque desde la economía
}

\section{MOOC courses: a view from the economy}

\author{
Nuria G. Rabanal \\ Universidad de León (España)
}

\section{Resumen}

La introducción de las Tecnologías de la Información y las Comunicaciones (TIC) en la enseñanza a nivel universitario ha supuesto un imparable proceso de innovación en la enseñanza y la aparición de nuevas formas de aprendizaje. Uno de los últimos fenómenos de aprendizaje ha sido el m-learning. Como una forma abierta y gratuita de transmisión del conocimiento global, este nuevo sistema de enseñanza ha tenido en los cursos MOOC (Massive Open Online Courses) el mejor ejemplo académico. El dinamismo de estos cursos y el numeroso grado de interactividad promovido han tenido su impacto en la forma de enseñar de las Universidades. Concebidos inicialmente como bienes libres accesibles a todos en cualquier sitio, estos cursos han promovido un debate en torno a su éxito y potencial beneficio económico. Aplicando los principios básicos de la teoría económica, nuestro trabajo hace una reflexión sobre el mercado de MOOC y los elementos determinantes de su demanda y oferta desde una perspectiva económica. El mercado de cursos MOOC se perfila como un mercado de bienes económicos públicos cercano a la competencia perfecta en sus características en el que la demanda y oferta toman la forma de los bienes económicos públicos con base en criterios cualitativos como la calidad y el altruismo. El beneficio directo o indirecto de esos cursos se fundamenta en una nueva forma de negocio donde las fuentes de ingresos están diversificadas y son analizadas.

Palabras clave: economía; aprendizaje abierto; aprendizaje móvil.

\begin{abstract}
The introduction of Information and Communication Technologies (ITC) for teaching at university level has culminated in an unstoppable innovation teaching process which generates new learning ways. One of the last learning phenomena has been "m-learning". As an open and inexpensive way of transmitting knowledge, this new teaching system has in MOOCs (Massive Open Online Courses) the best academic example. The dynamism of these courses and the high level of student interaction are also having an impact in distance learning universities. Conceived as elements accessible to anybody from everywhere, these courses have promoted a debate on their success and potential economic profit. Applying the basic principles of economic theory, this article provides some reflexions about the MOOC market and its offer and demand determinants from an economic point of view. Characteristics of MOOC market are quite similar to a certain competence markets. In addition, the demand and the offer in MOOC market have certain similarities with public goods and its functioning
\end{abstract}


depend on the interaction of variables like quality and altruism. The direct and potential indirect profits of these courses are the consequence of a new business way. Moreover, profit sources came from diversified sources and will be also analyzed in this paper.

Keywords: Economy; open learning; m-learning.

Es indudable que la incorporación de las TIC a los procesos de enseñanzaaprendizaje ha supuesto la aparición de ventajas educativas como la ampliación de la oferta formativa, la creación de entornos más flexibles para el aprendizaje o la eliminación de las barreras espacio-temporales para la interacción profesor-alumno o alumno-alumno. La diversificación de nuevas modalidades de comunicación ha servido de estímulo para la creación de nuevos entornos interactivos que favorecen un aprendizaje independiente y colaborativo, siendo los cursos MOOC un claro ejemplo de ello.

El cambio introducido por la tecnología en la enseñanza es indudable. Quizás uno de los primeros síntomas de avance en este sentido lo supuso la aparición del e-learning. El e-learning (Schneckenberg, 2004) impulsó la investigación que acompaña la necesaria búsqueda de nuevos modos de presentar el conocimiento que tienen lugar a partir de las innovaciones tecnológicas y que facilitan el acceso al mismo de los alumnos con independencia espacio-temporal. Con su surgimiento, se abrió una ventana de oportunidad para todos los agentes implicados en el proceso de aprendizaje al confluir los intereses de los potenciales demandantes con los empresariales, el deseo de encontrar nuevas aplicaciones para las innovaciones tecnológicas y la necesidad creciente de los centros educativos de disponer de fuentes adicionales de financiación. El resultado de este proceso ha tratado de conseguir que la educación sea un producto rentable para todos ellos.

Pero todo parece indicar que dentro de este fenómeno ha surgido una nueva forma de conocimiento globalizado que se ha alejado de los paradigmas clásicos del discutible pero cierto "negocio educativo": el m-learning y los cursos MOOC. Asumiendo que su conceptualización admite varias acepciones (Korucu y Alkan, 2011), está clara la connivencia inseparable entre aprendizaje y tecnologías móviles cuando se habla del m-learning, pudiendo relegar a un segundo plano las matizaciones que pueden hacerse en relación al grado de interactividad entre ambos aspectos o las maneras de manifestarse estos.

La literatura académica se ha esforzado también en conocer los matices que diferencian una y otra forma de aprendizaje realizando una comparación muy efectiva y concreta que pone de manifiesto las sutiles pero claras diferencias entre una y otra modalidad de enseñanza. En síntesis (Laouris y Eteokleous, 2005), las principales diferencias entre ambas modalidades pueden resumirse en que frente al e-learning, el m-learning es una forma de organización de la enseñanza más informal que la propia del e-learning. Los fines del aprendizaje difieren -algo evidente- en el caso 
que centra nuestro interés los cursos MOOC en los que los resultados del learning analytics $^{1}$ evidencian (Ho Reich, Nesterko, Seaton, Mullaney, Waldo y Chuang, (2014). HarvardX and MITx:, 2014; Torres Mancera y Gago Saldaña, 2014) que no es la búsqueda de formación académica y mejora de la cualificación formal la principal motivación en los alumnos de estos cursos, sino la enorme versatilidad y flexibilidad que este ofrece junto con una oferta formativa diversificada. La sustitución de elementos multimedia de alta resolución propios del e-learning por objetos dentro de aplicaciones y la interacción a través del aprendizaje colaborativo por la difusión a través del uso de redes son otros elementos diferenciadores.

La introducción del estadio evolutivo que comporta el m-learning ha supuesto, por tanto, cambios sustanciales en los instrumentos y en las tecnologías utilizadas para hacerlo viable y también en el diseño de los contenidos, implicando un cambio tecnológico y metodológico importante (Fini, 2009) capaz de proporcionar el soporte técnico necesario que permita acceder al conocimiento a través de estos nuevos soportes.

Es el uso generalizado de las nuevas herramientas de comunicación en la enseñanza derivadas de la incorporación de las TIC lo que nos lleva a las siguientes reflexiones: ¿seguimos haciendo lo de antes, pero de manera nueva o estamos aprovechando las nuevas tecnologías para hacer cosas nuevas de modos nuevos? ¿El modo en cómo se incorporan al proceso hace que se trate de un nuevo modelo de enseñanza-aprendizaje consolidable o de una moda pasajera? ¿Es esta nueva forma de aprendizaje incompatible con las tradicionales o no?

La búsqueda de respuestas a estas preguntas ha motivado una agitada discusión (Aguaded y Medina Salguero, 2015) en torno a los cursos MOOC y todo lo que implican. Lo cierto es que es un fenómeno que, lejos de convertirse en moda pasajera, se está consolidando como una forma nueva de conocimiento global.

La universidad española no ha permanecido ajena a esos cambios y junto con proyectos muy consolidados en modelos de e-learning está desarrollando proyectos dirigidos a un mercado potencial, el hispano hablante, que ha sido descuidado en su oferta formativa por las plataformas anglosajonas. El m-learning se ha consolidado de manera indiscutible con los cursos MOOC, incrementando de manera muy significativa la conectividad del aprendizaje (Kop, Fournier, y Mak, 2011). Considerados como un paso más hacia la idea de compartir de forma global el conocimiento, estos cursos tuvieron su predecesor en la Open Course Ware (OCW), herramienta pensada para ofrecer en abierto todo el material docente y académico elaborado por profesores universitarios. Nacidos, al igual que la OCW en Estados Unidos, los MOOC trasladan a un plano más formal la OCW generando con ello una auténtica revolución en la globalización del conocimiento.

En el universo de la red, ya son muchas las plataformas que ofertan este tipo de cursos y algunas de ellas acumulan una experiencia consolidada. Aunque en un primer momento se gestaron al amparo de las Universidades, su éxito ha permitido que se consoliden como entidades independientes, aunque ligadas al 
conocimiento universitario. Los proyectos más sólidos generados en universidades norteamericanas son ya conocidos por toda la comunidad educativa. En Europa los intentos siguen siendo menos sólidos y parecen tener un menor empuje, quizás debido a que el mercado europeo es menos flexible y competitivo, además de está más regulado que el norteamericano.

\section{LOS MOOC COMO BIENES PÚBLICOS}

El concepto de curso MOOC nace inicialmente pensado para que sea un conocimiento abierto y gratuito - massive and open- rasgos que inducen a alejar conceptualmente cualquier posibilidad de negocio en sentido estricto, llegando por intuición inicial que algo que es accesible a cualquier individuo y gratuito es un bien libre ${ }^{2}$.

Sin embargo, desde el punto de vista económico, no pueden considerarse como tales (Aguaded, 2013), ya que la existencia de restricciones tanto en su oferta como en su demanda plantean un problema de escasez que los convierte en bienes económicos. No solo su existencia no es ilimitada, sino que la demanda potencial está condicionada, entre otros, por factores tecnológicos.

Asumiendo, por tanto, que los cursos MOOC pueden ser considerados como bienes económicos -es decir sujetos a la norma de la escasez y los principios de utilidad y beneficio- y teniendo en cuenta las convenciones propias de la teoría económica, el "mercado" de cursos MOOC se acercaría en su evolución en el tiempo más a un modelo competitivo que monopolístico. Por definición, la competencia es tanto más pura cuanto mayor es el número de precio-aceptantes en un mercado, menores son las barreras, menor es la capacidad de que los oferentes influyan en la determinación del precio y más perfecta es la información para los que participan de él. Está claro que cumplen en gran medida estas premisas ya que el destino de estos cursos es un mercado global del conocimiento, con una oferta y demanda potencialmente universales, donde no existe posibilidad de establecer más barreras que las que se derivan del coste asociado a su puesta en marcha y la accesibilidad a Internet y, con poca influencia en los precios cuando estos no responden a la norma común del mercado. Por tanto, podemos hablar de la existencia de un modelo de negocio (O'Connor, 2014) y de un mercado académico en torno a los cursos MOOC.

Una vez superada la idea de que los cursos MOOC son bienes económicos, el siguiente paso es la consideración de si ese bien económico es público o privado. De acuerdo con la doctrina convencional (Samuelson, 2007), se dice que un bien económico es público cuando el consumo por parte de un individuo no limita el consumo por parte de los demás y privado si no se da esta condición. En este sentido, lo cursos MOOC cumplen con el requisito de que su demanda o uso por parte de un estudiante no limita el uso que hacen los demás, es más, los datos prueban que no hay límite de acceso a estos cursos en cuanto a número de estudiantes. Pero, además, el bien público tiene como característica adicional que el que se admita 
sea suministrado tanto por empresas privadas como públicas, teniendo que ver por tanto su consideración de público o privado más con la propia naturaleza del bien que con el tipo de institución o empresa que lo produce. La realidad es que estos cursos fueron inicialmente suministrados por empresas privadas -esencialmente universidades- y en la actualidad, cuando el mercado se ha hecho más grande, conviven simultáneamente universidades privadas y públicas.

Por otra parte, está la percepción del bien público por los usuarios de los mismos. La teoría económica diferencia igualmente entre los bienes públicos puros de los que están sujetos a congestión, es decir, cuyo exceso de demanda por parte de los usuarios acaba perjudicando su uso, siendo necesario establecer para ellos algún criterio de exclusión. En este sentido, los cursos MOOC se ajustan a este principio, pudiendo considerarse el acceso a internet o la necesidad de disponer de un ordenador el elemento excluyente. La exclusión garantiza en los bienes públicos menos ineficiencias que en un sistema competitivo lo que asegura la supervivencia en su provisión.

\section{EI PAPEL DEL ALTRUISMO EN EL MERCADO DE MOOC}

Conceptualmente, la oferta implica la creación de un producto con la finalidad de satisfacer una necesidad. Es decir, un empresario percibe una necesidad y decide satisfacerla mediante la producción de un bien bajo el principio de que este busca la maximización del beneficio con su provisión.

La oferta de MOOC fue liderada, como ya es sabido, por una única universidad que decidió en un momento determinado satisfacer la necesidad que muchos estudiantes tenían por aprender de una institución académica con reconocimiento a nivel mundial. Su experimento tuvo tal respuesta que, lo que en principio se pensó casi como un experimento sociológico, acabó por ser el comienzo de un fenómeno social y económico. El éxito inicial bien podría hacernos pensar en la formación de un mercado monopolístico ${ }^{3}$ e incluso oligopolístico con un claro liderazgo de sus precursores y otros centros de excelencia. Sin embargo, ha sido su caracterización como bien público excluyente con ausencia de barreras significativas de entrada al mercado junto a la versatilidad de las nuevas tecnologías la que ha hecho que la oferta se enriquezca en número con la creciente participación de oferentes. Desde entonces hasta ahora la expansión ha sido imparable.

Pero, puesto que nuestro análisis es desde la economía, cabe plantearse ¿Son los factores que determinan la oferta de MOOC equiparables a los que sigue cualquier otro bien económico?

Cualquier función de producción contempla necesariamente la combinación de factores productivos-capital y trabajo- y el elemento tecnológico, de manera tal que siguen en su comportamiento las reglas del juego de la teoría económica general. Lo que diferencia la oferta de MOOC de la oferta de otros productos similares es la eficiencia y eficacia en el uso combinado de todos ellos. Los cursos MOOC tienen una 
clara intensificación del uso del factor tecnológico haciendo que el factor variable más influyente en la determinación de los costes y del precio, el trabajo, tenga un bajo peso específico.

El factor trabajo cuenta en este tipo de cursos con una característica que solo se da con la provisión de bienes públicos y es que su participación en la función de producción está fuertemente determinada por una variable cualitativa: la de altruismo, mecenazgo, voluntariado o compromiso social del propio factor humano en la función de oferta. Ha sido este hecho el que ha contribuido igualmente al bajo precio implícito del factor variable.

El rol del altruismo y su papel en la determinación del equilibrio del mercado de los bienes ha sido ampliamente estudiado en la literatura económica (Davies y Lundholm, 2012; Echazu y Nocetti, 2015; O'Flaherty, 2003)due to the free rider problem, a theory of charitable giving based exclusively on altruism cannot explain the levels of contributions and participation observed in the data. In contrast, in this paper we demonstrate that altruism may have more predictive power than previously understood. We present a model in which the object of altruism is a large number of potential recipients of a good for which there is a target level of provision (e.g. an acute malnutrition treatment, an insecticide-treated bed net, tanto desde la perspectiva de cómo se perciben estos en el mercado, como la motivación desde la finalidad que persiguen con sus acciones quienes hacen de la generosidad su forma de participar en el mercado o la percepción que los consumidores (Isaac, Walker y Williams, 1994) tienen acerca del precio y si este ha de ser excluyente o no en un bien público.

No existe una teoría definitiva que explique de manera adecuada cómo los bienes públicos llegan a cubrir su oferta con acciones voluntarias cuando son muchos los individuos involucrados en la provisión del bien público. Las teorías más convencionales intentan explicar el comportamiento de los oferentes que voluntariamente toman parte de la prestación de un bien público a través de la maximización de la utilidad y de acuerdo a las conjeturas de Nash, lo que implica problemas en cuanto a la obtención del equilibrio general. Sin embargo, hay quienes proponen que este comportamiento se explica por el principio de reciprocidad (Sugden, 1985, p. 5, 2012) as some economists have argued, resolved \u2014 by relaxing the assumption of Nash conjectures. If individuals' conjectures are consistent, each person will hold the correct. $\mathrm{Si} G$ es el cualquier grupo de profesores del cual es miembro $i$ y suponemos que cada miembro de $G$ excepto $i$ está haciendo un esfuerzo $X$ en la realización de un curso, entonces i no puede hacer menos que ese esfuerzo $X$. La utilidad de cada individuo $U_{\mathrm{i}}$ en la provisión del bien público es función creciente de la cantidad de bien público que provee $Z$ y función decreciente del esfuerzo que realiza ${ }^{4}$. La realidad es que los cursos MOOC cumplen esa regla dado que quienes ofertan estos cursos contribuyen con su trabajo a aumentar la oferta del bien público, pero al mismo tiempo su función de utilidad decrece cuando el esfuerzo a realizar es mayor. 
La combinación de los elementos propios de la oferta de bienes públicos con un bajo coste marginal ha traducido los cursos MOOC en un producto con importantes economías de escala, lo que explica que un pequeño aumento en la dotación de factores implique aumentos mayores proporcionalmente en el producto final, lo que supone incorporar un efecto multiplicador a la oferta.

\section{BARRERAS Y MERCADO DE MOOC}

Tal y como hemos señalado anteriormente, el mercado de MOOC se aproxima al funcionamiento de un mercado de competencia perfecta. Está asumido en la comunidad económica que el modelo de competencia perfecta es eso, un modelo, no siendo posible encontrar en la realidad esta forma de mercado en estado puro, pero sí fórmulas que se asemejan en su comportamiento al modelo teórico.

La principal restricción por el lado de la oferta viene dada por la necesidad de una dotación mínima de factores productivos, sin la cual no es posible su puesta en marcha y que puede dar al bien MOOC la condición de excluyente de manera que esa exclusión favorecería la eficiencia de su mercado por tratarse de un bien público, pero impediría el acceso ilimitado al mercado. La disponibilidad de internet es la más importante ya que sin ella no podría canalizarse la oferta y no existiría demanda. La brecha tecnológica que se ha abierto a nivel mundial entre quienes disponen mayor y menor capacidad de $\mathrm{I}+\mathrm{D}+\mathrm{i}$ se ha traducido en una barrera de entrada al conocimiento abierto (Rohs y Ganz, 2015) ya que su no disponibilidad de conexión a internet junto con la carencia de cierta capacidad informática determina el acceso de los demandantes al mercado. La existencia de grandes diferencias en la dotación tecnológica, además, ha segmentado el mercado entre oferta y demanda. La oferta al igual que la demanda está lideradas por las Universidades de los países catalogados como desarrollados, no existiendo hasta la actualidad oferta procedente de países con bajos niveles de desarrollo o renta. Sin embargo, son estos segundos los que sí tienen una presencia en la demanda como muestran los resultados ${ }^{5}$ (Ho Reich, Nesterko, Seaton, Mullaney, Waldo y Chuang, (2014).

La asunción del dominio anglosajón por el lado de la oferta permite hablar igualmente de ciertas posiciones dominantes, pero también de una clara fragmentación que ha terminado por beneficiar el potencial de nuevas iniciativas dirigidas al mercado hispanohablante. Algunos autores (Rohs y Ganz, 2015) ven en el dominio lingüístico del inglés una barrera que actúa de manera excluyente. La ausencia de competencia inicial en el mercado hispanohablante ha potenciado el éxito de proyectos como MiriadaX, plataforma liderada por la Fundación Universia y la Fundación Telefónica, y COMA, de la Universidad Nacional de Educación a Distancia (UNED) que han visto y aprovechado un nicho de mercado, el hispanohablante, sin reacción inicial de las grandes plataformas anglosajonas. Aunque ambas plataformas establecieron al comienzo de su puesta en marcha una estrategia colaborativa, tras un año y medio de rodaje sin llegar a forzar la ruptura de la colusión necesaria para 
sobrevivir en los inicios de un proyecto de estas dimensiones, en la actualidad se configuran como dos proyectos diferenciados. MiriadaX cubre la captación de oferta proveniente de las Universidades Españolas y Latinoamericanas basadas en la docencia presencial pero que se lanzan a la enseñanza en abierto, mientras que COMA, el proyecto liderado por la UNED, concentra todo el potencial y las ventajas competitivas y economías de escala propias de la única Universidad Pública española de enseñanza a distancia con una experiencia acumulada que ha sido la esencia de su existencia, una infraestructura muy consolidada y desarrollada ${ }^{6}$ y con grandes ventajas en cuanto a implantación a nivel mundial.

Lo cierto es que en la actualidad la barrera lingüística ha sido resuelta con el propio desarrollo del mercado ya que el altruismo de muchos usuarios con conocimientos multilingües atendiendo la llamada al voluntariado desde las plataformas de estos cursos, permite disponer de transcripciones y subtítulos en varios idiomas. En otros casos, la disponibilidad de software específico y perfeccionado ha permitido hacer esta labor.

Junto al carácter o no competitivo en sentido convencional del "mercado" de este tipo de enseñanzas, encontramos una demanda potencial muy grande que se ve limitada por el acceso tecnológico a la red y la disponibilidad de la tecnología móvil necesaria. Los datos del Banco Mundial (Banco Mundial, 2014) muestran un progresivo y reciente crecimiento en el uso de internet en los países con una renta per-cápita media baja, lo que evidencia moderados síntomas de cambio, pero la brecha tecnológica sigue siendo enorme en relación a los países más desarrollados.

Sin embargo y a pesar de los datos, está claro que, si bien no se han suprimido todas las barreras al acceso al conocimiento, son accesibles de superar a medio plazo si se dan las condiciones necesarias para ello. Los costes comparativos revelan una clara ventaja de los cursos MOOC respecto de la enseñanza formal al permitir que un solo alumno de un país subdesarrollado con conexión a internet sea capaz de acceder al conocimiento de un centro de excelencia situado al otro lado del planeta que de otra manera sería imposible para él.

La asociación de una marca a la calidad implícita del producto por ella ofertado, juega un papel fundamental en el desarrollo del mercado. La calidad no es un concepto estandarizado y, aunque ha habido intentos por cualificar y cuantificar la calidad de estos cursos (Aguaded y Medina-Salguero, 2015; Ramírez-Fernández, 2015), lo cierto es que la calidad es un concepto subjetivo, sobre todo, cuando se trata de buscar datos cuantitativos que apoyen o refuten parámetros cualitativos. No obstante, los intentos tímidos realizados en esta línea muestran una correlación aceptable entre lo que cualitativamente se percibe y cuantitativamente se intenta probar respecto de su calidad.

Para los usuarios el mero hecho de poder acceder al conocimiento impartido por los centros más prestigiosos a nivel mundial ha supuesto un paso de gigante en la globalización del conocimiento y una mejora de la calidad de los recursos humanos que de ello se benefician, ya que siguen siendo los cursos liderados por instituciones 
prestigiosas las que lideran la demanda. Aunque a la motivación de la calidad puedan unirse otras razones (Al-Atabi y Deboer, 2014; Roscoria, 2014), la búsqueda de nuevas experiencias o ganar en independencia adquiriendo nuevos conocimientos, el acceso a la calidad a bajo coste es sin duda un elemento determinante.

Tomando como referencia algún ejemplo (O’Flaherty, 2003) que analiza las especificidades que rodean la determinación del equilibrio en un mercado de bienes basados en la gratuidad, podríamos establecer ciertas similitudes sobre la caracterización del mercado de los MOOC y el comportamiento de los bienes gratuitos y su calidad.

Los demandantes reaccionan positivamente ante mejoras de la calidad y viceversa. Los cambios en la demanda vienen provocados por el cambio en la necesidad que pueden experimentar los alumnos en la ordenación de sus preferencias, de manera que un cambio en las preferencias positivamente genera un cambio de la demanda positivo también. Por el lado de la oferta, si asumimos que estamos en un mercado más próximo al de competencia perfecta, también la calidad determina esta ya que se considera paramétricamente como un elemento determinante. En este caso los cambios en la oferta responden a cambios en el grado de generosidad implícita a quienes los ofrecen.

Esta situación evidencia que cualquier cambio en cómo perciben los potenciales usuarios la calidad ofrecida, cambios en las preferencias de estos o el establecimiento de posibles precios no vinculados a la calidad pueden incidir en el equilibrio del mercado.

\section{LA BÚSQUEDA DEL BENEFICIO}

Hablar de mercado y hacerlo desde una perspectiva económica nos lleva necesariamente a la búsqueda del beneficio. El beneficio económico queda determinado por la diferencia entre los ingresos y los costes. Si el precio lo determina la calidad, pero no se traduce en un valor monetario, ¿Es posible hablar de beneficio económico en estos cursos? Si es así ¿dónde se localiza ese beneficio?

Si atribuimos a los cursos MOOC la condición de bienes económicos públicos, el beneficio es un beneficio social no tangible, es decir, para quienes han diseñado y desarrollado estos cursos gratuitamente su satisfacción radica en la satisfacción derivada de su generosidad (Andreoni, 1988). Para los usuarios o demandantes los beneficios son los posibles rendimientos en cuanto a su propia mejora del conocimiento y formación.

Si partimos del hecho inicial de que la educación es sin duda un producto rentable (Schneckenberg, 2004) y asumiendo que las innovaciones técnicas provocan un cambio estructural profundo, ya nadie duda de que estos cursos a pesar de su gratuidad han generado un nuevo modelo de negocio y, por tanto, de ingresos y beneficios. Aunque inicialmente están concebidos como cursos gratuitos, es decir, cualquier persona puede acceder a los contenidos y actividades de los mismos, lo 
cierto es que su desarrollo en el tiempo ha venido acompañado de fórmulas de negocio que proporcionan un feedback económico para las instituciones y organizaciones que están detrás de la iniciativa. Alentadas por la búsqueda de algo más que el mero hecho de mejorar el nivel de conocimiento global, los oferentes se han percatado de que existe un potencial de negocio indirecto - pero no por ello menos lucrativodetrás de su puesta en marcha y no incompatible con el altruismo y beneficio social que les inspira.

Una primera fuente de ingresos es el derivado de la propia infraestructura tecnológica necesaria para acceder a este mercado. Aunque existen varias vías de rentabilizar el uso de las plataformas (Belleflamme y Jacqmin, 2016; Jansen y Schuwer, 2015), nos centraremos en los beneficios ligados directamente con el usuario final más que en el intermedio derivado de la provisión del servicio. En este sentido, las compañías de telecomunicaciones son las primeras beneficiadas de un fenómeno que ha hecho que millones de usuarios intensifiquen no solo el consumo de dispositivos móviles, sino también el de datos en internet siendo para ello necesario acceder a un segmento de consumo por encima del mero uso del teléfono móvil para llamadas. Según los últimos datos del INE en España el número de usuarios se ha incrementado notablemente en sus distintas formas. Además, la incorporación del m-learning se traduce en un uso más necesario de internet y de su capacidad. $\mathrm{Si}$ hasta hace unos años el mayor número de móviles no aumentaba el mismo ritmo que el número de usuarios con datos móviles de internet, ahora esta tendencia se ha invertido.

Junto al beneficio directo que supone hablar de las telecomunicaciones está el de la publicidad. Muchos cursos utilizan canales como YouTube, que son ventanas a la publicidad y difusión que proporcionan ingresos adicionales en las plataformas. Lo mismo ocurre con las redes sociales, utilizadas por muchos cursos como actividad para fomentar el networking e incluso la empleabilidad de los cursos por no decir la publicidad. Pero no solo la publicidad directa proporciona ingresos. Muchas universidades ven en la realización de este tipo de cursos un medio muy eficaz para captar candidatos a cursos en enseñanza reglada cuya demanda muchas veces no prospera porque, si bien el prestigio del centro suele considerarse suficiente, el coste económico de la formación oficial en muchos de estos centros es tan alto que el potencial cliente quiere asegurarse accediendo a una versión low-cost, de que realmente valdrá la pena pagar el dinero que exige realizar un curso reglado en cualquiera de estas prestigiosas universidades y la universidad oferente ve en ello una inversión rentable a medio plazo. Pero la publicidad no solo se circunscribe a estas formas convencionales, los resultados editoriales de plataformas y materiales que, de otra manera, no serían conocidos por la comunidad global también tienen aquí su canal de difusión y pueden favorecer la financiación de nuevos proyectos.

Un tercer beneficio radica en que el diseño del acceso a estos cursos, provee a las plataformas educativas que gestionan este conocimiento de una base de datos inmensa en la que los alumnos aportan datos de su perfil (edad, sexo, nivel de 
estudios, situación geográfica etc...), lo que permite un conocimiento detallado del demandante y una segmentación personalizada de la publicidad, así como establecer perfiles sobre potenciales receptores de información. Los datos de los alumnos no solo proporcionan un canal de publicidad para el propio proyecto académico, sino que sirven para captar talentos y crear un mercado interno de trabajo en el que empiezan a estar interesadas muchas empresas. La demanda de empleo a través de estas fórmulas está cobrando cada vez más importancia, hasta el punto que eso ha terminado por generar nuevos ingresos a través de fórmulas como la verificación de conocimientos.

La expansión de esta fórmula y su bajo precio han permitido que la propia concepción de curso "abierto" haya evolucionado hacia una vía de negocio basada en la acreditación de conocimientos y hacia el nacimiento del Corporate learning. $\mathrm{Si}$ inicialmente es cierto que la motivación estaba en la posibilidad de acceder al conocimiento impartido por centros excelentes, sin que la acreditación de dicho conocimiento fuese determinante para su demanda, a medida que se ha ido consolidando la idea de que lo gratuito puede tener calidad y que se ha constatado que estos cursos pueden mejorar la capacitación de los estudiantes mejorando su cualificación a bajo coste y permitiéndoles acceder a mejoras en el mundo profesional y laboral, han surgido sub-formulas evolucionadas de ingresos. Estas sub-fórmulas se basan esencialmente en certificación de conocimientos. Las plataformas como COURSERA han establecido simultáneamente a la versión gratuita y en abierto la posibilidad de acreditar - previo pago de una modesta cantidad y pruebas de evaluación y calificación más estrictas que en la versión gratuita- de manera oficial los conocimientos adquiridos. Esto ha provocado un giro en el mercado de los MOOC radical. Una vez constatado el éxito de la fórmula y que se ha conseguido el reconocimiento del mercado laboral a la calidad académica y de formación a través de estos cursos, se ha puesto en marcha recientemente una nueva propuesta: la especialización curricular. La fundación SAYLOR, fue la primera precursora de esta fórmula al poner en marcha auténticos grados sin reconocimiento académico siguiendo la estructura de cursos en abierto. Sin embargo, ha sido COURSERA la que de manera verificada ha empezado a dar forma a esta especialización basada en MOOC. Por tanto, convive paralelamente la forma verificada con la gratuita. Tratándose del mismo producto, en un caso el alumno por un bajo precio puede acceder a una cualificación certificada.

El pago monetario de un precio por la certificación combinada con la posibilidad de adquirir una especialización permite hablar del tránsito de un mercado académico como el descrito -determinado en su comportamiento por un precio en calidad- a una fórmula de mercado más evolucionada, donde a la calidad y la verificación se suma la especialización. La forma de fidelizar clientes en este tipo de cursos se ha hecho a través de la especialización. Con una considerable mayor flexibilidad que la formación presencial y un coste reducido, los demandantes cursan varios cursos con certificado de verificación y obtienen un nuevo certificado como especialista. 
A través de este mecanismo se consiguen simultáneamente varios fines: satisfacer una demanda a bajo precio, proporcionar ingresos, mejorar la formación y fidelizar alumnos evitando un problema subyacente y a veces relacionado con su calidad como es la tasa de éxito.

Considerando que hemos dicho que la demanda es muy elástica ${ }^{7}$, cualquier precio por debajo del esperado de equilibrio normal del mercado, hará que se disparen los ingresos. Cualquier aumento de precio no acorde a lo esperado y en la media hará caer la matriculación. Básicamente, el diseño del mecanismo de precios en estos cursos parte del principio de que vender mucho a bajo precio proporciona más ingresos que vender caro a precios altos, un precepto económico que se cumple si y solo si la demanda es elástica (algo por otra parte coherente con el grado de competitividad). Los precios que se cobran por esta certificación son bajos, suelen rondar los $36 \$$ $-46 \$$ y los 50 euros los más bajos. Tomemos como referencia el curso de Harvard (Summary, 2015) con 181.000 alumnos, si asumimos que solo un $2 \%$ de los alumnos pagasen unos $45 \$$ por la certificación del curso, los ingresos ascenderían a162.900 \$ por un solo curso sin que ello haya supuesto excesivos costes adicionales ${ }^{8}$.

Pero esta estrategia no solo ha calado entre los alumnos que han visto la oportunidad de mejorar su cualificación, sino que -más importante que eso aúnlas propias empresas han dado el marchamo de calidad al admitir como mérito evaluable en un candidato el haber realizado cursos MOOC. De esta manera ha surgido el corporate learning (Overton, Dixon y Towards Maturity, 2014), que hace referencia a cómo las compañías o empresas a nivel internacional usan los MOOC para formar a su personal y también rentabilizan sus formaciones internas a través del diseño de sus propios MOOC con certificación. El empuje de la oferta recibido por los demandantes de empleo, es decir las empresas, ha dado un giro espectacular al potencial económico de estos cursos y favorecido que muchas universidades vean en los MOOC un recurso financiero adicional.

Por último, cabe señalar un beneficio implícito para el conocimiento y la investigación globales y que se deriva del uso del diseño metodológico de los cursos como laboratorio y centro de investigación. Un buen diseño metodológico de los ejercicios prácticos puede convertir un curso en un laboratorio sociológico y experimental del que obtener resultados para la investigación e innovación de aquellos centros que lo ofertan multiplicando las economías de escala derivadas de su puesta en marcha, proveyendo a los investigadores de una herramienta a bajo coste de la que obtener una alta rentabilidad científica. Pensemos por ejemplo en encuestas vinculadas a ejercicios donde los resultados de la muestra pueden alcanzar resultados hasta ahora impensables.

\section{CONCLUSIONES}

La educación del futuro ha de poder realizarse en cualquier momento, ejecutarse en cualquier lugar, ha de ser personalizable, estar adaptada a las necesidades de quien 
la recibe y contemplar un papel activo por parte del estudiante, que se convierte en el auténtico protagonista de su aprendizaje. El éxito de los cursos MOOC permite hablar de un nuevo modelo de aprendizaje que está teniendo, entre otras consecuencias, el que los cursos se planteen bajo demanda, se tienda a que estén operativos todo el año y la formación se conciba como un proceso continuo que acompaña al individuo toda su vida y se transmita una información reutilizable.

El éxito alcanzado por los cursos MOOC permite hablar de su definición como bienes económicos sujetos más a las normas de un mercado de competencia perfecta que a otras formas de mercado. Al igual que en cualquier mercado, la oferta y demanda y su libre juego determinan su éxito, encontrándonos con que la calidad del curso es un factor esencial que juega un papel semejante al del precio de los bienes en un mercado. No solo se trata de bienes económicos, sino que son bienes económicos públicos excluyentes cuyo comportamiento en la oferta viene explicado por el altruismo y el principio de reciprocidad. La demanda tiene como elementos determinantes los beneficios sociales recibidos y una buena relación calidad-precio y como elementos excluyentes el acceso a la tecnología móvil necesaria.

El mercado de los MOOC permite hablar de nuevas formas de negocio y por tanto de beneficio diferentes a las usadas en la enseñanza más tradicional. Estas nuevas fórmulas de negocio con bajos precio, pero con un reconocimiento cada vez mayor del mercado laboral ratifican la rentabilidad subyacente. Para cumplir con los requisitos y reglas del juego de este mercado será indispensable basar la estrategia en la calidad del conocimiento ofertado, y como se trata de un mercado competitivo, será el libre juego de oferta y demanda basada en este criterio el que a largo plazo "filtrará" el éxito del modelo emprendido por cada Universidad. El éxito de esta fórmula no radica en la copia de una estructura fija. Muchas universidades, especialmente las españolas, han visto en estos cursos una fórmula exclusivamente económica, basando su estrategia en la copia en "versión española" de la estructura metodológica y didáctica de los cursos anglosajones, dejando de lado la calidad. A medio plazo, la exclusión lingüística que permite el estar dirigidas al mercado hispano hablante les proporciona una ventaja competitiva que deberían saber aprovechar para reconducir su estrategia.

\section{NOTAS}

1. Con la expresión learning analytics hacemos referencia al término anglosajón comúnmente aceptado para referirse al análisis de datos de aprendizaje obtenidos en cursos MOOC en función de las encuetas y también los datos aportados por las plataformas informáticas sobre descargas de contenidos números de "cliks" realizadas por los alumnos de estos cursos. Nosotros asumiremos el término anglosajón a lo largo del texto.

2. La teoría económica convencional diferencia entre los bienes libres y los bienes económicos. 
3. El monopolio se caracteriza por ser un mercado opuesto en sus características a la competencia y donde se dan en su extremo opuesto todas las características de un mercado de competencia perfecta: un solo oferente (caso del monopolio de oferta) y muchos demandantes, capacidad por tanto para influir en el precio, información imperfecta y barreras de entrada y salida al mercado.

4. Es decir, que para un profesor la utilidad es mayor cuanto mayor es el número de cursos ofertados pero esa misma utilidad decrece cuando es esfuerzo es mayor.

5. Los datos obtenidos en este informe han establecido el punto de origen a través de la localización geográfica de la IP del usuario de los cursos.

6. La UNED cuenta con canal propio de televisión y radio, y con centros asociados en medio mundo.

7. La elasticidad precio de la demanda es un concepto que explica por qué vender a bajos precios puede proporcionar mayores ingresos que vender ese mismo producto a precios elevados.

8. Básicamente es casi nula la inversión de recursos adicionales para garantizar la acreditación que el estudiante solicita ya que los mecanismos de evaluación son los mismos que para el alumno en matrícula libre, solo cambia el control en la ejecución de las actividades programadas y/o la exigencia de una nota mínima.

\section{REFERENCIAS BIBLIOGRÁFICAS}

Aguaded, J. I. (2013). The MOOC Revolution: A new form of education from the technological paradigm? Comunicar, 41, 7-8. doi: http://doi.org/10.3916/C412013-a1

Aguaded, J. I., y Medina Salguero, R. (2015). Criterios de calidad para la valoración y gestión de MOOC. RIED: Revista Iberoamericana de Educación a Distancia, 18(2), 119-143.

Al-Atabi, M., y Deboer, J. (2014). Teaching entrepreneurship using Massive Open Online Course (MOOC). Technovation, 34(4), 261-264. Recuperado de http:// www.scopus.com/inward/record. url?eid=2-s2.0-84896784898\&partnerID $=40 \&$ md $5=8 \mathrm{~b} 4$ fo $717888 \mathrm{bbc} 47 \mathrm{bb} 1 \mathrm{ce} 7 \mathrm{c} 23$ $844 \mathrm{bd} 67$

Andreoni, J. (1988). Why free ride?: Strategies and learning in public goods experiments. Journal of Public Economics, 37(3), 291-304. doi: http:/ doi.org/10.1016/0047-2727(88)90043-6

Banco Mundial. (2014). Abonados a internet por banda ancha fija por cada 100 personas. Recuperado de http://datos. bancomundial.org/indicador/IT.NET. BBND.P2/countries/1W?display=default Belleflamme, P., y Jacqmin, J. (2016). An economic appraisal of MOOC platforms: Business models and impacts on higher education. CESifo Economic Studies, 62(1), 148-169. doi: http://doi. org/10.1093/cesifo/ifvo16

Davies, P., y Lundholm, C. (2012). Students' understanding of socio-economic phenomena: Conceptions about the free provision of goods and services. Journal of Economic Psychology, 33(1), 79-89. doi: http://doi.org/10.1016/j. joep.2011.08.003

Echazu, L., y Nocetti, D. (2015). Charitable giving: Altruism has no limits. Journal of Public Economics, 125, 46-53. doi:http:/ doi.org/10.1016/j.jpubeco.2015.03.002

Fini, A. (2009). The Technological Dimension of a Massive Open Online Course: The Case of the CCKo8 Course Tools. International Review of Research in Open and Distance Learning, 10(5). 
Ho Reich, J., Nesterko, S., Seaton, D. T., Mullaney, T., Waldo, J., y Chuang, I. (2014). HarvardX and MITx: The first year of Open Online Courses. MIT office of Digital Learning. Recuperado de http:// ssrn.com/abstract $=2381263$

Isaac, M. R., Walker, J. M., y Williams, A. W. (1994). Group size and the voluntary provision of public goods: Experimental evidence utilizing large groups. Journal of Public Economics, 54, 1-36. doi:http:/ doi.org/10.1016/0047-2727(94)90068-X

Jansen, D., y Schuwer, R.(2015).Institutional MOOC strategies in Europe. Recuperado de http://www.futurelearninglab.org/wpcontent/uploads/2015/03/www.eadtu eu documents Publications OEenM Institutional MOOC strategies in Europe.pdf

Kop, R., Fournier, H., y Mak, J. S. F. (2011). A Pedagogy of Abundance or a Pedagogy to Support Human Beings? Participant Support on Massive Open Online Courses. International Review of Research in Open and Distance Learning, 12(7), 74-93.

Korucu, A. T., y Alkan, A. (2011). Differences between m-learning (mobile learning) and e-learning, basic terminology and usage of m-learning in education. 3rd World Conference on Educational Sciences - 2011, 15(0), 1925-1930. doi: http:// doi.org/http://dx.doi.org/10.1016/j. sbspro.2011.04.029

Laouris, N., y Eteokleous, Y. (2005). We need an educationally relevant definition of Mobile Learning. Recuperado de http:/ www.mlearn.org.za/CD/papers/Laouris \& Eteokleous.pdf

O'Connor, K. (2014). MOOCs, institutional policy and change dynamics in higher education. Higher Education, 1-13. Recuperado de http://www.scopus. com/inward/record.url?eid=2-s2.084894283790\&partnerID $=40 \& \mathrm{md} 5=\mathrm{fbg}$ 6a3e8fc8fff2d9113b61079c17cb6

O'Flaherty, B. (2003). Need and generosity: how markets for free goods equilibrate.
Journal of Urban Economics, 54(1), 157172. doi: http://doi.org/10.1016/So0941190(03)00025-1

Overton, L., Dixon, G., y Towards Maturity. (2014). Using MOOCs to transform traditional training. Recuperado de www.towardsmaturity.org/in-focus/ MOOC2014

Ramírez-Fernández, M. B. (2015). La valoración de MOOC: una perspectiva de Calidad MOOC appraisal: A quality perspective. RIED. Revista Iberoamericana de Educación a Distancia, 18(2), 171-195.

Rohs, M., y Ganz, M. (2015). MOOCs and the Claim of Education for All : A Disillusion by Empirical Data. International Review of Research in Open and Distributed Learning, 16(6), 1-19.

Roscoria, T. (2014). 3 Reasons why people take massively open online courses. Recuperado de http://www. centerdigitaled.com/policy/WhyMassively-Open-Online-Courses.htm

Samuelson, P. A. (2007). The Pure Theory of Public Expenditure Paul A. Samuelson. The Review of Economics and Statistics, 36(4), 387-389.

Schneckenberg, D. (2004). El "e-learning" transforma la educación superior. Educar, 33, 143-156. Recuperado de http://dialnet.unirioja.es/servlet/ extart?codigo $=989408$

Sugden, R. (1985). Consistent conjectures and voluntary contributions to public goods: why the conventional theory does not work. Journal of Public Economics, 27(1), 117-124. doi:http://doi. org/10.1016/0047-2727(85)90032-5

Sugden, R. (2012). Reciprocity : The Supply of Public Goods Through Voluntary Contributions. Economic Journal 94(376), 772-787.

Summary, E. (2015). HarvardX and MITx : Two Years of Open Online Courses.

Torres Mancera, D., y Gago Saldaña, D. (2014). Los Moocs y su papel en la 
creación de comunidades de aprendizaje y participación Participación. RIED. Revista Iberoamericana de Educación a Distancia, 17(1), 13-34. doi: http://doi. org/10.5944/ried.17.1.11570

\section{PERFIL ACADÉMICO Y PROFESIONAL DE LA AUTORA}

Nuria González Rabanal. Profesora del Departamento de Economía y Estadística de la Universidad de León y directora del grupo GiDUM de innovación docente desde donde ha desarrollado numerosos proyectos de innovación docente universitaria: Cursos híbridos, Student Response Systen en el aula, gamificación, flipped classroom, entre otros... Fundadora del Teaching \& Learning Innovation Institute (TeLe(In)2) destinado a promover la innovación docente en el ámbito universitario, es presidente del mismo desde 2014.

E-mail: nuria.grabanal@unileon.es

\section{DIRECCIÓN DE LA AUTORA}

Universidad de León

Facultad de Ciencias Económicas

Departamento de Economía y Estadística

Campus Vegazana s/n 24007

León (España)

Fecha de recepción del artículo: 30/05/2016

Fecha de aceptación del artículo: 07/08 /2016

\section{Como citar este artículo:}

González Rabanal, N. (2017). Cursos MOOC: un enfoque desde la economía. RIED. Revista Iberoamericana de Educación a Distancia, 20(1), pp. 145-160. doi: http://dx.doi.org/10.5944/ried.20.1.16664 